\title{
Tether tension performance utilizing various materials for a coplanar and non-coplanar model of a flexible tethered satellite system
}

\begin{abstract}
This paper presents a numerical model for a flexible tethered satellite system in both planar and co-planar environment. This tethered satellite system consists of three rigid bodies with two flexible tethers, each connecting two rigid bodies with one located in the center serving as the mothership. The dynamics of the system include tether deformations, rotational dynamics and orbital mechanics. Five different materials that are commonly used will be tested accordingly in order to observe its performance based on the tension of the tether. It is found that, based on all of the materials simulated, diamond has the best tension performance.
\end{abstract}

Keyword: Coplanar model; Non-coplanar model; Tether material; Tethered satellite systems 